\title{
A Vision for Co-optimized T\&D System Interaction with Renewables and Demand Response
}

\author{
C. Lindsay Anderson \\ Cornell University, USA \\ cla28dcornell. edu
}

\author{
Luckny Zéphyr \\ Cornell University, USA \\ lz395@cornell. edu
}

\author{
Judith B. Cardell \\ Smith College, USA \\ jcardellesmith.edu
}

\begin{abstract}
The evolution of the power system to the reliable, efficient and sustainable system of the future will involve development of both demand-and supply-side technology and operations. The use of demand response to counterbalance the intermittency of renewable generation brings the consumer into the spotlight. Though individual consumers are interconnected at the low-voltage distribution system, these resources are typically modeled as variables at the transmission network level. In this paper, a vision for cooptimized interaction of distribution systems, or microgrids, with the high-voltage transmission system is described. In this framework, microgrids encompass consumers, distributed renewables and storage. The energy management system of the microgrid can also sell (buy) excess (necessary) energy from the transmission system. Preliminary work explores price mechanisms to manage the microgrid and its interactions with the transmission system.

Wholesale market operations are addressed through the development of scalable stochastic optimization methods that provide the ability to co-optimize interactions between the transmission and distribution systems. Modeling challenges of the co-optimization are addressed via solution methods for large-scale stochastic optimization, including decomposition and stochastic dual dynamic programming.
\end{abstract}

Index Terms-renewable energy, microgrid, responsive demand, stochastic optimization

\section{Introduction}

Power system operation involves scheduling generating technologies, differing in capacity and capability, to meet demand that is distributed across the network. The risk and uncertainty associated with system operation increases with the introduction of new generation and consumer-based technologies. Additionally, system operation is constrained by line power transfer capabilities, generator minimum and maximum output limits, ramping capabilities, and the probability of contingencies. The integration of intermittent renewable generating technologies increases the uncertainty in the operation of the network (adding to existing uncertainty from demand, and generator and line outages, for example). The introduction of consumer-based (distributed) generation and the increasing presence of microgrids further affect the historical net-load patterns that utilities have relied upon for planning purposes. Stochastic optimization offers a rigorous method to address optimal management in power system economic dispatch, unit commitment, and investment planning processes.

The uncertainty and variability of renewable power generation can be characterized through sophisticated resource forecasting followed by modeling the aggregated power output from wind farms and solar fields. However, it is difficult to accurately represent the underlying stochastic processes of renewable resources. In addition to the mathematical challenge, inadequate representation of uncertainty can lead to costly or infeasible decisions based on erroneous information [1]. Other technologies in the power system must be available for mitigating the varying power output from intermittent renewables.

Initial efforts to balance variability or renewable resources have relied upon varying the output of conventional generating technologies. More recently, responsive demand is being included as an important network resource that can mitigate renewables' variability. Responsive demand has the benefit of being distributed throughout the network and being capable of rapid deployment, as well as requiring little additional infrastructure. Due to these advantages, the use of responsive demand for uncertainty management has been explored in many recent works, including [2], [3], [4], [5], [6], [7], [8]. These projects share the commonality of integrating responsive demand and renewables into the transmission system operations (and have paid less attention to the distribution system level).

The power system modeling framework introduced in this paper relies upon the fact that a significant portion of flexible loads are managed within the energy management system of the distribution system, potentially organized into microgrids, in which commercial and residential consumers are interconnected with distributed generation. To date there has been little work in the co-optimization of the high- and low-voltage systems with renewables and responsive loads. Therefore, the vision presented here is the development of a comprehensive co-optimization framework that incorporates the generation and transmission system with microgrids that include responsive loads, distributed generation, intermittent generation and storage. The interaction of these two complex systems can be leveraged to manage the risk and uncertainty 
inherent in the future power system with a high penetration of intermittent and distributed resources.

The modeling and simulation framework has four elements. The first element focuses on developing multi-area renewable resource modeling methods that preserve both spatial and serial correlations. This element incorporates statistical- and optimization-based methods to generate scenarios for various classes of renewables, correctly correlated in space and time. The second element investigates demand response strategies to mitigate the impacts of renewables' intermittency on operation costs through micro-grid modeling, that integrates the energy management of the microgrid with the demand response strategies. The third element investigates the interaction between the low- and high-voltage grids to manage congestion in the network and alleviate the impact of intermittent renewables. This element incorporates microgrid dynamics with the operational models of the transmission grid, and investigates optimal locations and interactions for microgrids in terms of transmission system operation. The fourth element pulls from all elements in the framework to develop a comprehensive stochastic cooptimization framework that incorporates renewables, demand response and storage with judicious decomposition methods that ensure reliable solutions that are scalable to large networks.

\section{Background}

\subsection{Wholesale Electricity Markets for High- and Low-Voltage Systems}

The uncertainty and variability of renewables in the future electric grid will require new paradigms for system operation and management. The flexibility inherent in responsive loads is significant in enabling the use of these environmentally benign technologies. However, the majority of the existing research conducted on responsive loads has focused either on the transmission system or the distribution system. The framework presented here focuses on demand response integration at the distribution level, coordinated with operations at the transmission level, in order to best understand the efficacy of these resources and the various implementation programs under consideration.

Since the majority of renewable and traditional generation resources are controlled by independent system operators at the transmission level, it is critical to incorporate both levels of the power system into comprehensive analyses of the use of responsive demand for managing risk and uncertainty in the electric grid. The vision presented here is novel in that it proposes large-scale co-optimization of transmission- and distribution- level systems to allow accurate modeling of the technology interactions. Participation of microgrids and flexible distribution systems in the wholesale electricity market will be a significant and enabling structure for effective demand side participation.

Initial results are presented here and the broader framework is designed to facilitate the co-operative optimization of micro- and macro-grids. The framework will be tested via case studies on increasingly large networks, with larger networks representing different markets and regions. These networks will be chosen for various characteristics and for the purposes of testing scalability. The formulation and solution methods developed as part of this project will be generally applicable to different market structures across North America.

\subsection{Microgrids and the Smart Grid}

The power system is built upon the interconnections of generation, transmission and distribution, potentially organized as microgrids [9]. As continuous efforts are deployed to build robust, reliable and resilient power networks to mitigate the consequences of uncertain events, distribution networks, the starting point of a significant proportion of network failure, require special attention [9]. The future of the existing power grid relies on moving toward a selfhealing system accommodating a variety of supply- and demand-side technologies, including renewables and storage facilities. In addition, the electric power system is evolving due to pressures from smart grid technologies, restrictions on pollutant emissions and the need to maintain low overall cost. Distributed energy resources organized into microgrids will play an increasingly important role in this evolution through encouraging both greater installation of renewables, such as rooftop solar and residential-agricultural scale wind power, and also smart grid technologies such as smart meters and automated demand response, typically implemented at customer sites at low voltage levels [1], [10], [11], [12], [13], [14], [15]. The vision presented here assumes that the low-voltage system will be operated as a microgrid, with smart grid technologies, to enable demand response and coordination of distributed generation.

\section{Framework for Optimizing the Future Power System}

The overarching objective of the modeling and analysis framework presented here is the development of a scalable co-optimization solution for transmission and distributionsystem-as-microgrid, that includes demand response, storage and renewable resources. This solution incorporates modeling and integration of responsive demand in the low voltage system to assist in management of uncertainty in the transmission system induced by renewable generation sources and contingencies. To achieve this objective, the framework includes four overlapping elements:

1) Develop new optimization and statistical approached to properly characterize the uncertainty, spatial correlation, and serial correlation of renewables, including solar and wind.

2) Identify and develop viable strategies and models for integrating demand response in the low voltage grid, and evaluating potential application to mitigating risk and uncertainty in the grid. 


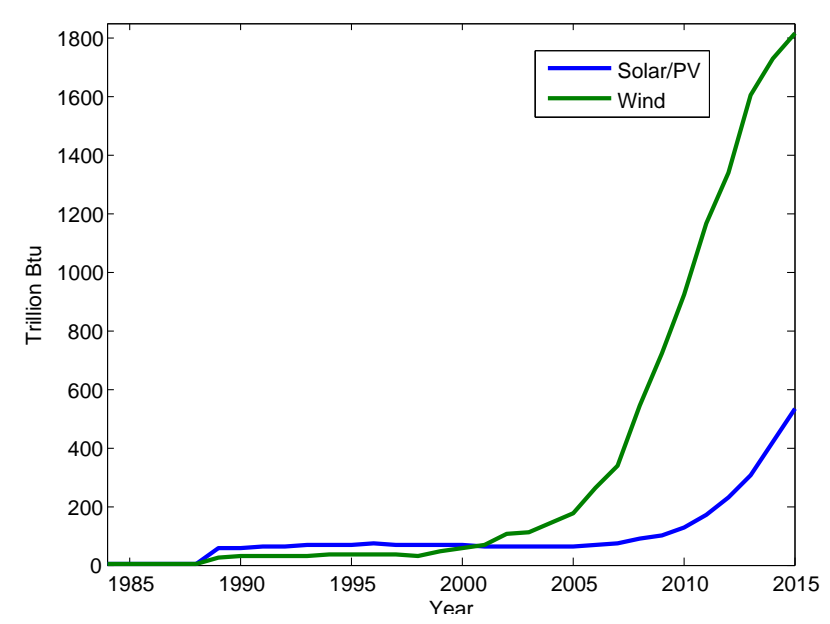

Figure 1. Solar/PV and wind consumption in the US from 1984 to 2015.

3) Incorporate elements 1 and 2 to examine the interaction between the high-voltage and low-voltage systems, with the objective of identifying operating strategies that improve the performance of the combined, unified systems.

4) Building upon the first three elements, develop an overall stochastic co-optimization framework that includes transmission and distribution systems with renewables, demand response, and storage capabilities, and that is scalable to systems of any size.

\subsection{Element 1: Multi-area renewable resources modeling with spatial and serial correlations}

Wind and solar/PV consumption in the US has been increasing since the beginning of the twenty-first century as depicted in Figure 1 (data from [25].) In 2015, renewable energy sources contributed about $13 \%$ to the total US energy generation and accounted for about $10 \%$ of total consumption. This indicates an important step towards the $20 \%$ renewable energy integration perspective by 2030 . The increasing renewable penetration, however, further complicates the operation of the power grid as it introduces new sources of uncertainty and variability in its management, requiring more sophisticated decision support tools be developed.

The first element of the the framework focuses on innovative methods to characterize the uncertainty in renewable generation with specific focus on incorporating the spatial and temporal correlations that are typically not well characterized in existing methods.

Accurate renewable modeling is crucial as forecasting errors may result in costly operating decisions. Accurate models should take into consideration the correlation structure, since renewable resources may be correlated across areas because of similar meteorological conditions. Several methods have been reported for wind speed/wind power forecasting [26], [27], [28]; however, none of the existing research has compared the performance of these methods. In addition, other methods widespread in other areas of stochastic programming [29], [30], [31] are not effectively applied for renewable resources. Consistent with [32], the modeling vision includes development of forecasting/simulation methods based on non-linear optimization. Initial investigations in this area are presented in Section 4.

The framework also recognizes the need to empirically compare the performance of existing and alternative methods. One step in this element is to investigate statistical and optimization-based methods to generate spatially and serially correlated renewable scenarios. Algorithms and methods comparisons on different problems and data sets will provide conclusive insights, as an algorithm can outperform another on some data sets and underperform on others. The greater the number of tests included in testing, the more valid the conclusions. An important second focus is to perform numerical experiments with various data sets (for different locations).

\subsection{Element 2: Microgrid DR strategies to mitigate intermittency in the transmission system}

Previous work by the authors developed a mechanism to facilitate significantly increasing distributed resource penetration in the power system by coordinating their participation in electricity markets [21], [24], [33]. This framework expands upon the earlier work by explicitly including intermittent generation resources on the supply-side and improving the sophistication of the demand response resources through integration with the work done in [2]. The increased intelligence in power system facilities as the smart grid continues to develop suggests that modeling these facilities as intelligent agents is consistent with their actual implementation. In addition, software running the devices that will constitute microgrids and smart grid installations is itself increasingly agent-based, supporting the modeling and simulation of microgrid facilities as intelligent software agents. The framework discussed here integrates prior work into the earlier modeling framework developed by the authors, focusing on co-optimization of transmission- and distribution-level operations.

This second element thus focuses on the modeling of demand-side resources in the low voltage network. This element builds on previous work on agent-based methods in [21], [24], [33], explicitly including intermittent generation resources on the supply-side and improving the sophistication of the demand response resources. This element also explores alternative strategies to incorporate demand response resources and develop novel demand response strategies and models through microgrid modeling.

\subsection{Element 3: Synergistic interactions and strate- gies for the high-voltage- and micro-grids}

The joint optimal and cooperative operation of the distribution-system-as-microgrid with the high-voltage 
power network requires new modeling representations of the unified system. In-depth understanding of technology and system interactions is crucial in developing a joint optimization model that captures the dynamics of the unified system. A first step is determining which optimization approach(es) best fit the cooperative operation of the unified system. This, for example, may translate into a single objective, or a multi objective or bi-level optimization problem.

Element three considers the interaction between the micro and macro grids to develop strategies for co-operative optimization of these systems to optimize performance of the entire power system. The approach includes analysis of interactive effects, development of potential strategies to encourage effective interaction, and assessment of the impact of microgrid location on the transmission system.

Within Element 3, the effort includes developing an optimization framework that captures the microgrid dynamics as well as its interaction with the high voltage network. This effort to develop co-optimization framework will contribute to the open question as to where to best locate microgrid, and to incent effective interactions within the unified system. This is envisioned to be addressed within a broader perspective while tackling specific issues facing the bulk power system such as congestion management.

\subsection{Element 4: A comprehensive stochastic co- optimization framework for the future power sys- tem}

The power system co-optimization framework introduced here unifies the high-voltage transmission network with interconnected microgrids. The microgrids contain the interconnected intermittent renewable generating technologies, responsive demand, and storage. The framework analysis discussed here increases the size and complexity of the power network computational problem, building upon the strengths of efficient stochastic optimization methods and algorithmic schemes. Several optimization algorithms have been developed since the 1970 s in an attempt to solve large scale problems. Some of those algorithms are limited to specific classes of problems while others have a broader application scope. Problem structure provides insights on strategies to tackle computational burden. For instance, in a day ahead market structure, the decision making is divided into two steps, specifically (i) the on/off status of the conventional generators (the unit commitment decisions), and (ii) the dispatch level of each committed unit as well as their contribution to reserve requirements (the economic dispatch decision). When the power network also comprises storage facilities, economic dispatch also addresses the strategic utilization of storage over the planning horizon, increasing the decision space. The strategy for the framework presented here is to investigate decomposition algorithms to address the tractability of the problem.

This final element incorporates the lessons from the other elements in the development of the co-optimization framework that integrates the transmission and microgrid level systems. It is well known that realistic transmission level optimization problems are of high dimension and computationally challenging, and the addition of the low voltage subsystems will exacerbate the complexity. Therefore significant effort focuses on the use of decomposition strategies for the unit commitment aspect of the problem, and the application of stochastic dual dynamic programming (SDDP) to the economic dispatch stages. This element leads to a solution framework that models the interactive and co-operative effects of the microgrid/distribution system managing demand-side resources with the larger transmission system, including large scale intermittent renewables. This approach is significant as the majority of demand-side resources will exist in the low voltage network, and realistic management of these resources must include these systems explicitly.

As discussed earlier the operation of the transmission network in itself is complicated due to transmission constraints and risk of failure. Therefore, the stochastic co-optimization frame is challenging, even for relatively modest size networks, supporting the development of a comprehensive stochastic optimization framework to tackle the global problem. As computer capability has increased, stochastic optimization methods, not equality efficient, have been proposed. Of particular interest here are decomposition techniques. These methods, in conjunction with efficient algorithms, hold promise for solving large scale problems.

\section{Related Prior Work}

The purpose of the modeling framework and future view for the power system presented here is to develop a structured and scalable solution to incorporate responsive demand, storage and intermittent renewables within the high and low-voltage networks in an efficient and reliable manner.

The project builds on the authors' previous experiences in development of innovative methods for scalable stochastic optimization of power systems [2], [16], [17], and the modeling interactions between renewables and the existing grid [1], [18], [19]. Previous work conducted by the authors, both individually and collaboratively, have focused on the use of current market structure to enable integration of renewables and responsive loads in the future electric grid [1], [18], [19], [20]. The following sections discuss related prior work that supports the development of this framework.

\subsection{Renewables Integration}

In the area of renewables integration, [1] focuses on the potential for leveraging interactions of wind and demand response. [19] investigates renewable variability on the efficacy of the EPA's Clean Power Plan to target existing generation and [18] presents an empirical analysis of integration strategies for wind self-procurement of reserves, referred to as a flexible dispatch margin. As the level of wind penetration increases, the impacts of the uncertainty associated with wind generation become more significant on system operations. The flexible dispatch margin is designed 
to make wind generation available when moving from the hour-ahead to the 10-minute market.

In providing a flexible dispatch margin, wind generators under-schedule in the hour ahead energy market in order to hold some of their expected, forecasted, output in reserve, in the day-ahead schedule. This schedule is submitted for less than the forecasted wind generation, providing expected reserves for mitigating system variability and uncertainty. The expected excess wind generation is then available for mitigating forecast errors from wind and demand as well as lost generation from forced outages.

Fig. 2 depicts possible 10-minute market realizations with the use of a flexible dispatch margin, FDM, for wind generation. The far left bar shows an hour-ahead forecast of 100MW for some wind farm, with the lower (blue) portion of $90 \mathrm{MW}$ representing the schedule submitted as the wind farm schedule in the hour-ahead market. The remaining 10 MW (upper green segment) represents the flexible dispatch margin held as expected reserves for the 10-minute market.

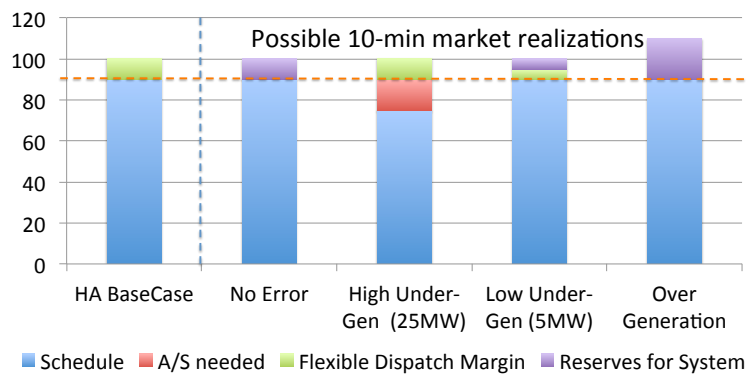

Figure 2. 10-minute market realizations with a wind flexible dispatch margin.

The four right-hand bars in Fig. 2 show possible 10minute market realizations. The 'no error' bar is the situation in which the 10-minute ahead forecast is the same as the hour-ahead forecast, resulting the hour-ahead schedule of $90 \mathrm{MW}$ being met, and leaving $10 \mathrm{MW}$ of expected wind generation available to the system. In the 'high undergeneration' situation, the wind generation is forecasted to be only $75 \mathrm{MW}$. In this case the wind farm imposes the need on the system for $15 \mathrm{MW}$ of additional generation, while 10 MW of the otherwise $25 \mathrm{MW}$ under-generation is accounted for through the FDM. For the 'low under-generation' case, the wind is now forecasted to generation $95 \mathrm{MW}$, meaning that $5 \mathrm{MW}$ from the FDM are used to mitigate the wind farm forecast error from the hour-ahead time stage yet leaving 5 MW available to the system as needed. Finally for the 'over generation' case in which the wind farm is now expected to generate $110 \mathrm{MW}$, there will be $20 \mathrm{MW}$ of wind generation expected to be available for the system if needed.

Simulated use of a wind flexible dispatch margin has been shown to increase system-wide benefits including less generator ramping, lower LMP and fewer price spikes [18]. The flexible dispatch margin has the potential to improve power system performance in terms of decreased reliance on peaking generators to mitigate the increased net variability and uncertainty. Integrating the flexible dispatch margin for wind into the framework presented in this paper will require optimizing the magnitude of the flexible dispatch margin for different levels of wind penetration and system demand.

\subsection{Distributed Resources in Microgrids}

The authors have previously investigated modeling and control of distributed generation and microgrid operations [21], [22], [23], [24], and the mobilization of responsive demand and distributed generation [2], [21], [23], [24].

Distributed resources, both responsive demand and distributed generators, are at a disadvantage in wholesale electricity markets due to their relatively small size (as well as existing market rules). Organized as microgrids, these distributed resources can potentially participate in electricity markets, on both the generation and demand side. As discussed throughout this paper, the power system will require a new framework for coordinating system and market operations as the number of market participants grows. Rules for participation in ancillary services markets vary regionally, yet tend to include similar requirements for facilities and associated communications capabilities, increasingly enabled by smart grid technologies. Price signals are one mechanism available to coordinate the operation of a power system in the emerging competitive environment.

In ideal markets, the market clearing dynamic relies upon the actions of both customers and suppliers as they respond to price changes according to their own price elasticities. Price elasticity is defined as the percent change in quantity (demand or supply) for a given percent change in price. Typically, a price increase would cause suppliers to increase their output while customers would decrease their demand in order to avoid paying the higher price. Distributed resource response based strictly upon individual and isolated decisions is acceptable for distributed resource participation in bulk energy markets. However, this dynamic could result in a net energy imbalance within a closed microgrid system as the number of these resources grows and if resources respond strictly in an open loop manner, based on their independently determined price elasticity.

Building upon the concept of frequency droop, one mechanism designed to prevent an energy imbalance and possible instability is the concept of price droop [24]. The price droop is a method for coordinating market behavior and facilitating distributed resource participation in the local balancing market, and presenting the microgrid response as a unified participant in the wholesale markets. Patterned after the definition of frequency droop, price droop is defined to be the percentage change in price for a given percentage change in quantity.

$$
\text { PriceDroop }_{i}=\frac{\triangle \lambda / \lambda_{0}}{\triangle P_{i, G / L} / P_{i, G_{0} / L_{0}}}
$$

where $\lambda$ represents the system price and $P_{i, G / L}$ represents either generator input or load consumption. $\Delta \lambda$ is the change in system spot price from a given system disturbance, $\triangle P$ is the response of the generator or load to the system spot 


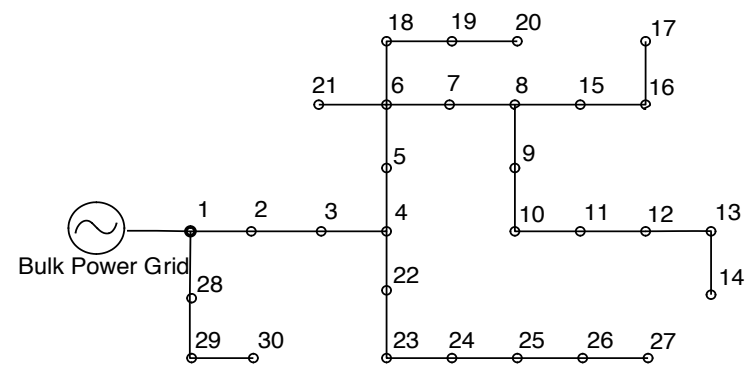

Figure 3. 31 bus test system

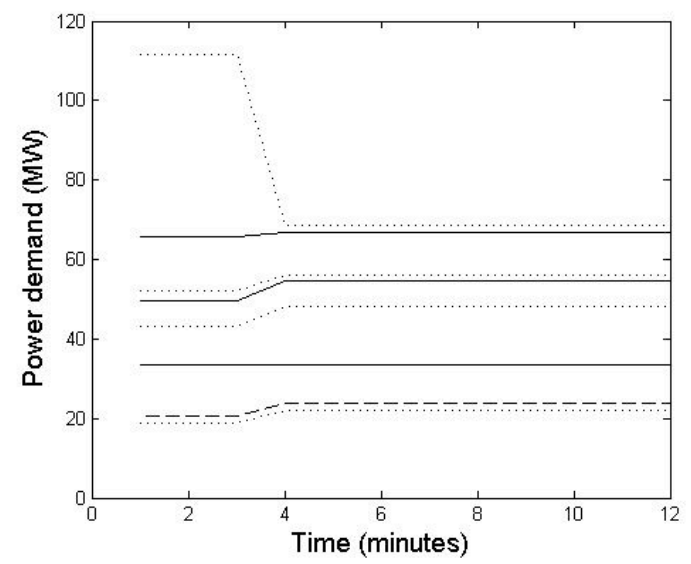

Figure 4. Responsive load behavior after a load disturbance of -50MW and subsequent local price change, based on price droop values learned through Q-learning [33].

price, $\lambda_{0}$ represents the initial system price, and $P_{i, G_{0} / L_{0}}$ represents the maximum load or generation capacity.

Initial results for implementing the price droop mechanism in a microgrid where all participating distributed resources are modeled as intelligent agents are presented below. The 31 bus test system used for the simulations is shown in Fig. 3. Generators are located at buses 10, 17 and 24 with load at all the remaining buses except for buses 1 , $3,5,6,7,8$.

For a given operating point, price droop values for all participating resources can be calculated in a coordinated fashion, which will ensure cooperative behavior. This mechanism could be particularly useful in microgrids that are dominated by facilities interconnected via power electronics, and are thus without synchronized generators with large inertia to provide the traditional frequency droop.

For a load disturbance of a decrease in 50MW of demand, figures 4 and 5 show the response of other customers and distributed generators, respectively, as coordinated by the price droop mechanism. These figures demonstrate that in response to an initial drop in load, the rest of the microgrid follows a proportional response in which the generators decrease their output and the loads increased their consumption slightly in order to maintain the local energy balance.

This initial investigation with the price droop mechanism

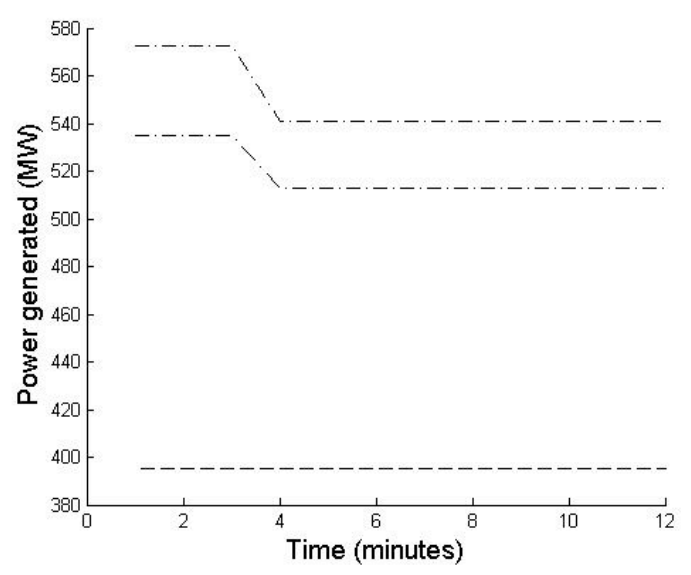

Figure 5. Distributed generator response to a local load disturbance and subsequent price change, based on price droop values learned through Qlearning [33].

demonstrates the integration of distributed energy resources into electricity markets and reliable system operations. The price droop mechanism an open-loop, multi-agent signal that builds upon the concepts of frequency droop and own price elasticity. These simulations using the signal demonstrate the ability of distributed energy resources, organized into microgrids and coordinated to act as a unified entity, to participate in electricity markets while maintaining system reliability.

The price droop signal is anticipated to be useful in promoting increased use of distributed energy resources, including demand response and intermittent renewable energy technologies, and is integrated into the larger framework presented in this paper.

\subsection{Microgrid and Transmission System Interac- tions}

Preliminary work described in [23] explores interaction between microgrid and the transmission system through a simulation study conducted on the IEEE 30 bus test system. The microgrid includes demand response, storage capability, and solar generation resources, while the transmission system includes thermal generation and wind resources. In this work, the interaction between the microgrid energy management policy, and the economic dispatch of the transmission system are considered. The key parameters considered are the location of the microgrid, as well and the pricing policy governing the interaction between the connected systems.

Specifically, results show that transmission system congestion can be alleviated or exacerbated through the addition of a microgrid at specific nodes on the system. In addition, the pricing policy for purchase (sales) of energy by the microgrid from (to) the transmission system shows significant potential for the management of congestion conditions. For example, Figure 6 shows the likelihood of line congestion under various pricing schemes guiding microgrid/transmission grid interaction. 


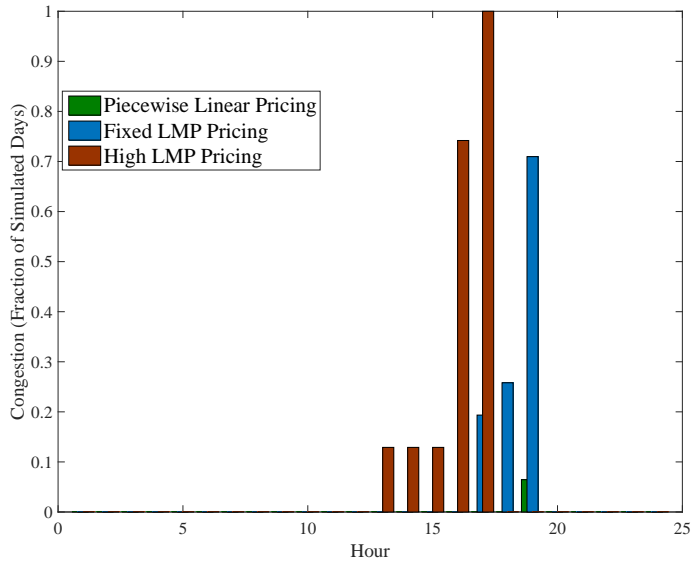

Figure 6. Likelihood of transmission congestion under alternative pricing schemes between microgrid and transmission system, shows significant sensitivity

In Figure 6, three distinct pricing mechanisms are considered; a fixed LMP-based (FLMP) strategy, wherein the microgrid pays (receives) a fixed price for purchase (sale) of energy at the transmission level, based on the economic LMP. The congestion results are also compared with a high (fixed) LMP strategy (HLMP), as well as a piecewise linear price function (PWL), based on a priori sensitivity analysis of the network. It is clear from examination of Figure 6, that the pricing mechanism plays an important role in managing the interaction between the microgrid and the transmission system, and can be leveraged to encourage mutually beneficial behavior.

It is through this combination of previous work that it has become apparent that a truly comprehensive approach for the management of the future grid will include microgrids, distribution systems, and the transmission system in a co-optimization approach.

The framework described here follows a natural evolution to combining this previous work for developing methods and solutions for the co-operative optimization of the transmission system and the distribution-systemas-microgrid. This framework is essential to leverage the consumer-level demand response, toward management of a power system with significant renewable resources. The vision is based on knowledge of the complexities and dimensionality of the systems involved, and the optimization framework is formulated to leverage decomposition methods and approximation (SDDP) approaches for ensuring computational tractability.

\section{Closing Remarks}

An ideal microgrid is defined as an electric entity capable of operating in both interconnected (with the highvoltage grid) and islanded mode. As such, the microgrid should incorporate generating units (e.g. traditional units and intermittent) and if needed, absorb (feed) power from (to) the high-voltage grid. The interplay between the microgrid and high-voltage grids drives the interest in developing the co-optimization approach to ensure performance of the global network.

Demand-side participation cannot be leveraged effectively without explicitly including the distribution system dynamics in the optimization-based wholesale market operations. To date, very little research has been conducted on the co-optimization of these two systems due to computational limitations. However, advances in computational capabilities, and the judicious use of decomposition methods and innovative approximation methods for high-dimension dynamic programming (SDDP) now makes this goal a viable objective that will lead to a fundamental shift in the integration of demand-side resources.

To this end, the modeling framework presented introduces a novel co-optimization framework to include the operations of both the transmission and distribution systems (or microgrid) to effectively analyze renewable and distributed generation along with responsive demand.

This vision for the future power system relies upon: i) a performance comparison of renewable output forecasting/scenario generation methods, ii) a comprehensive framework for the interplay between the micro and macro grids, iii) an analysis of the impacts of different demand response strategies on the power grid operation, and finally iv) an analysis of the performance of combined stochastic decomposition methods to tackle the integrated problem.

Previous work by the authors supports this vision as a natural evolution for the integration of synergistic technologies for the achievement of reliable, economic and sustainable energy for the future.

\section{Acknowledgement}

This work was supported in the National Science Foundation, grant numbers IIA-1243482 (the WINDINSPIRE project), NSF SEP-1230788, and NSF ECCS-1453615.

\section{References}

[1] J. Cardell and C. Anderson, "Analysis of the system costs of wind variability through monte carlo simulation," in 2015 48th Hawaii International Conference on System Sciences. IEEE, 2015, pp. 1-8.

[2] J. Liu, M. Martinez, B. Li, J. Mathieu, and C. Anderson, "A Comparison of Robust and Probabilistic Reliability for Systems with Renewables and Responsive Demand," in Proceedings of the 49th Hawaii International Conference on System Sciences (HICSS). IEEE, 2016, pp. 2373-2380.

[3] M. Vrakopoulou, J. L. Mathieu, and G. Andersson, "Stochastic Optimal Power Flow with Uncertain Reserves from Demand Response," in Proceedings of the 47th Hawaii International Conference on System Sciences (HICSS). IEEE, 2015, pp. 2353-2362.

[4] Y. Zhang and G. B. Giannakis, "Distributed Stochastic Market Clearing With High-Penetration Wind Power," IEEE Transactions on Power Systems, vol. 31, no. 2, pp. 895-906, Apr. 2016.

[5] Kris Ruud, "Wind Forecast Integration at MISO," http://www.ieeepes.org/presentations/td2014/td2014p-000699.pdf. 
[6] Robert Ethier, "Integration of Renewables into the Electric System: Opportunities and Challenges," http://www.iso-ne.com/staticassets/documents/2015/10/clg-robert-ethier-panel-presentation-10-92015-final.pdf.

[7] Solar Industry Staff, "CAISO Plans Upgrades To Boost Renewable Energy Management," http://solarindustrymag.com/caiso-plansupgrades-to-boost-renewable-energy-management.

[8] California ISO, "Building a Sustainable Energy Future: 20142016 Strategic Plan,” https://www.caiso.com/Documents/20142016StrategicPlan-ReaderFriendly.pdf.

[9] H. Farhangi, "The path of the smart grid," Power and energy magazine, IEEE, vol. 8, no. 1, pp. 18-28, 2010.

[10] R. Lasseter, A. Akhil, C. Marnay, J. Stephens, J. Dagle, R. Guttromson, A. S. Meliopoulous, R. Yinger, and E. J, "Integration of distributed energy resources: the certs microgrid concept," CERTS Report, April, 2002.

[11] Electric Power Research Institute, "Smart grid demonstration initiative (updated january 2015)," Available: http://smartgrid.epri.com/Demo.aspx.

[12] European Microgrid Consortium, Available: http://microgrids.power.ece.ntua.gr/micro/index.php .

[13] A. Sfetsos, "A comparison of various forecasting techniques applied to mean hourly wind speed time series," Renewable energy, vol. 21, no. 1, pp. 23-35, 2000.

[14] IRED (Integration of Renewable Energy Sources and Distributed Generation into the European Electricity Grid), Available: http://www.ired-cluster.org/ .

[15] NYSERDA, "Ny prize competition," Available: http://www.nyserda.ny.gov/All-Programs/Programs/NY-Prize.

[16] G. Martinez and L. Anderson, "A risk-averse optimization model for unit commitment problems," in System Sciences (HICSS), 2015 48th Hawaii International Conference on. IEEE, 2015, pp. 2577-2585.

[17] M. Martinez and C. L. Anderson, "Toward a scalable chanceconstrained formulation for unit commitment to manage high penetration of variable generation," Allerton Conference on Communication, Control and Computing, pp. 1-8, 2014.

[18] J. Cardell and C. Anderson, "A Flexible Dispatch Margin for Wind Integration," IEEE Transactions on Power Systems, pp. 1-8, May 2014.

[19] — "Targeting existing power plants: EPA emission reduction with wind and demand response," Energy Policy, vol. 80, pp. 11-23, May 2015.

[20] _ " "The Influence of Demand Resource Response Time in Balancing Wind and Load," in 2013 46th Hawaii International Conference on System Sciences (HICSS). IEEE, 2013, pp. 2364-2370.

[21] C. Y. Tee and J. Cardell, "Market Integration of Distributed Resources Through Coordinated Frequency and Price Droop," IEEE Transactions on Smart Grid, vol. 5, no. 4, pp. 1556-1565, Apr. 2016.

[22] J. Cardell and M. Ilic, The Control and Operation of Distributed Generation in a Competitive Electric Market. Springer US, 1998, ch. 12, pp. 451-516, iSBN 978-1-4419-5038-3.

[23] J. Liu, M. Martinez, and C. Anderson, "Quantifying The Impact Of Microgrid Location And Behavior On Transmission Network Congestion," in Proceedings of the 2016 Winter Simulation Conference. IEEE, 2016, p. 11, under Review.

[24] J. B. Cardell, "Distributed resource participation in local balancing energy markets," in Power Tech, 2007 IEEE Lausanne. IEEE, 2007, pp. $510-515$.

[25] US Energy Information Administration, "US wind and solar consumption from 1984 to 2015," http://www.eia.gov/totalenergy/data/monthly/index.cfm\#renewable.
[26] M. Lei, L. Shiyan, J. Chuanwen, L. Hongling, and Z. Yan, "A review on the forecasting of wind speed and generated power," Renewable and Sustainable Energy Reviews, vol. 13, no. 4, pp. 915-920, 2009.

[27] S. S. Soman, H. Zareipour, O. Malik, and P. Mandal, "A review of wind power and wind speed forecasting methods with different time horizons," in North American Power Symposium (NAPS), 2010. IEEE, 2010, pp. 1-8.

[28] A. M. Foley, P. G. Leahy, A. Marvuglia, and E. J. McKeogh, "Current methods and advances in forecasting of wind power generation," Renewable Energy, vol. 37, no. 1, pp. 1-8, 2012.

[29] J. Dupačová, N. Gröwe-Kuska, and W. Römisch, "Scenario reduction in stochastic programming," Mathematical programming, vol. 95, no. 3, pp. 493-511, 2003.

[30] N. Gröwe-Kuska, H. Heitsch, and W. Römisch, "Scenario reduction and scenario tree construction for power management problems," in Power Tech Conference Proceedings, 2003 IEEE Bologna, vol. 3. IEEE, 2003, pp. 7-pp.

[31] K. Høyland, M. Kaut, and S. W. Wallace, "A heuristic for momentmatching scenario generation," Computational optimization and applications, vol. 24, no. 2-3, pp. 169-185, 2003.

[32] L. Söder, "Simulation of wind speed forecast errors for operation planning of multiarea power systems," in Probabilistic Methods Applied to Power Systems, 2004 International Conference on. IEEE, 2004, pp. 723-728.

[33] J. B. Cardell and C. Y. Tee, "Distributed energy resources in electricity markets: The price droop mechanism," 48th Annual Allerton Conference on Communication, Control, and Computing, pp. 58-65, 2010. 